\title{
Noncommutative Wormhole Solutions in Einstein Gauss-Bonnet Gravity
}

\author{
Shamaila Rani and Abdul Jawad \\ Department of Mathematics, COMSATS Institute of Information Technology, Lahore 54000, Pakistan \\ Correspondence should be addressed to Abdul Jawad; jawadab181@yahoo.com
}

Received 2 November 2015; Revised 25 January 2016; Accepted 3 February 2016

Academic Editor: Rong-Gen Cai

Copyright ( 2016 S. Rani and A. Jawad. This is an open access article distributed under the Creative Commons Attribution License, which permits unrestricted use, distribution, and reproduction in any medium, provided the original work is properly cited. The publication of this article was funded by SCOAP . $^{3}$

\begin{abstract}
We explore static spherically symmetric wormhole solutions in the framework of $n$-dimensional Einstein Gauss-Bonnet gravity. Our objective is to find out wormhole solutions that satisfy energy conditions. For this purpose, we consider two frameworks such as Gaussian distributed and Lorentzian distributed noncommutative geometry. Taking into account constant redshift function, we obtain solutions in the form of shape function. The fifth and sixth dimensional solutions with positive as well as negative GaussBonnet coefficient are discussed. Also, we check the equilibrium condition for the wormhole solutions with the help of generalized Tolman-Oppenheimer-Volkoff equation. It is interesting to mention here that we obtain fifth dimensional stable wormhole solutions in both distributions that satisfy the energy conditions.
\end{abstract}

\section{Introduction}

The study of wormhole solution becomes a prime focus of interest in the modern cosmology as it connects different distant parts of the universe as a shortcut. The wormhole is like a tunnel or bridge with two ends which are open in distant parts of the universe to join. To develop the mathematical structure of wormhole in general relativity [1], the basic ingredient is an energy-momentum tensor which constitutes exotic matter. This is hypothetical form of matter results of the violation of energy conditions. For two-way travel, the traversable condition must be fulfilled; that is, the throat of wormhole must remains open due to violation of the null energy condition. Since normal matter satisfies the energy conditions, the matter violating the energy condition is called exotic. The phantom dark energy violates the energy conditions which may be a reasonable source of wormhole construction $[2,3]$. The inclusion of some scalar field models, electromagnetic field, Gaussian and Lorentzian distributions of noncommutative geometry, thin-shell formalism, and so forth demonstrates more interesting and useful results [4-11].

In order to minimize the usage of exotic matter or to find another source of violation while normal matter satisfies the energy conditions, many directions are adopted so far. Modified theories of gravity are the most appealing direction which contribute to using effective energymomentum tensor. For instance, in $f(R)$ [12] as well as $f(T)$ [13] theories, it has been proved that the effective energy-momentum tensor which consists of higher order curvature terms or some torsion terms is responsible for the necessary violation to traverse through the wormhole. Nowadays, higher dimensional wormhole solutions are also under discussion. Many theories point out the existence of extra dimensions in the universe which leads to explore wormhole solutions for higher dimensions.

Rahaman with his collaborators have done a lot of work taking noncommutative geometry in four-dimensional spacetime as well as higher dimensional cases. Rahaman et al. [14] studied the higher dimensional static spherically symmetric wormhole solutions in general relativity with Gaussian distribution. They found these solutions up to four dimensions while they found these solutions in a very restrictive way for fifth dimensional case. Bhar and Rahaman [15] obtained the same result by taking Lorentzian distribution. Rahaman et al. [16] worked for viable physical properties of new wormhole solutions inspired by noncommutative geometry 
with conformal killing vectors. In $f(R)$ gravity with Gaussian [17] and Lorentzian distributions [8], wormhole solutions are constructed but with violation of energy conditions. Sharif and Rani [13] explored the noncommutative wormhole solutions in $f(T)$ gravity and found some physically acceptable solutions.

In a recent paper, Jawad and Rani [18] studied Lorentz distributed wormhole solutions in $f(T)$ gravity and found some stable wormhole solutions satisfying energy conditions. In the higher dimensional gravity theories, $n$-dimensional Einstein Gauss-Bonnet gravity is widely used. The modern string theory established its natural appearance in the low energy effective action. Bhawal and Kar [19] studied Lorentzian wormhole solutions in $D$-dimensional Einstein Gauss-Bonnet gravity which depend on the dimensionality of the spacetime and coupling coefficient of Gauss-Bonnet combination. Taking traceless fluid, Mehdizadeh et al. [20] extended this work and found wormhole solutions which satisfy energy conditions.

We explore wormhole solutions taking spacetime of $(n-2)$ sphere in the framework of $n$-dimensional Einstein Gauss-Bonnet gravity. We consider two frameworks: noncommutative geometry having Gaussian distributed energy density and Lorentzian distributed energy density. For $n=$ 5 and 6-dimensions, we take positive as well as negative Gauss-Bonnet coefficient. Also, we check the stability of wormhole solutions with the help of generalized TolmanOppenheimer-Volkoff equation. The paper is organized as follows: in Section 2, we construct the field equation for higher dimensional wormhole solutions in $n$-dimensional Gauss-Bonnet gravity. Section 3 is devoted to the construction of wormhole solutions in Gaussian and Lorentzian noncommutative frameworks. In Section 4, we check the equilibrium condition of the wormhole solutions. Section 5 summarizes the discussions and results.

\section{Field Equations}

In this section, we provide some basic and brief reviews about $n$-dimensional Einstein Gauss-Bonnet gravity as well as wormhole geometry and construct field equations in the underlying scenario.

2.1. n-Dimensional Einstein Gauss-Bonnet Gravity. The action for $n$-dimensional Einstein-Gauss-Bonnet gravity is an outcome of string theory in low energy limit. It is given by

$$
S_{n \mathrm{~GB}}=\int \sqrt{-g}\left[R-\epsilon_{1} \mathscr{L}_{\mathrm{GB}}\right] d^{n} x,
$$

where $R$ is the $n$-dimensional Ricci scalar, $\epsilon_{1}$ is the GaussBonnet coefficient, and $\mathscr{L}_{\mathrm{GB}}$ is the Gauss-Bonnet term defined as

$$
\mathscr{L}_{\mathrm{GB}}=R^{2}-4 R_{\alpha \beta} R^{\alpha \beta}+R_{\alpha \beta \gamma \delta} R^{\alpha \beta \gamma \delta} .
$$

Varying the action with respect to metric tensor, the field equations becomes

$$
G_{\alpha \beta}+\epsilon_{1} \mathbb{G}_{\alpha \beta}=\mathscr{T}_{\alpha \beta},
$$

where $G_{\alpha \beta}$ and $\mathscr{T}_{\alpha \beta}$ are the Einstein and energy-momentum tensors, respectively, while Gauss-Bonnet tensor is defined as

$$
\begin{aligned}
\mathbb{G}_{\alpha \beta}= & 2\left(R R_{\alpha \beta}-2 R_{\alpha \gamma} R_{\beta}^{\gamma}-2 R_{\alpha \gamma \beta \delta} R^{\gamma \delta}-R_{\alpha \delta \sigma \tau} R_{\beta}^{\sigma \tau \delta}{ }_{\beta}\right) \\
& -\frac{1}{2} \mathscr{L}_{\mathrm{GB}} g_{\alpha \beta} .
\end{aligned}
$$

It is noted that we assume $8 \pi G_{n}=1$ where $G_{n}$ is the $n$ dimensional gravitational constant.

2.2. Wormhole Geometry. The wormhole spacetime for $(n-2)$ sphere is given by $[14,15]$

$$
d s^{2}=-e^{2 \lambda(r)} d t^{2}+\frac{d r^{2}}{1-s(r) / r}+r^{2} d \Omega_{n-2}^{2},
$$

where $\lambda(r)$ is the redshift function and $s(r)$ is the shape function. For traversable wormhole scenario, we have to choose $\lambda$ to be finite to satisfy the no-horizon condition. Usually, it is taken as zero for the sake of simplicity, which gives $e^{2 \lambda(r)} \rightarrow 1$. The reason behind finite redshift function is as follows. The redshift function defines that part of the metric responsible for finding the magnitude of the gravitational redshift. The gravitational redshift is the reduction in the frequency that a photon will experience when it climbs out from gravitational potential well in order to escape to infinity. In doing so, the photon uses energy. Its energy is proportional to its frequency. A reduction in energy then is equivalent to a reduction in frequency, which is also known as redshift function. If the wormhole has an event horizon, it means that a photon emitted outwardly from the horizon cannot escape to infinity. In other words, it would take an infinite amount of energy for the photon to escape. Its frequency would be infinity reduced; that is, its redshift would be negatively infinite. A negatively infinite value of the redshift function at a particular value of the radial coordinate indicates the presence of an event horizon there. Thus to be traversable wormhole solution, the magnitude of its redshift function must be finite.

The shape of the wormhole is such that a spherical hole in space with increasing length of diameter as moving far from throat (the minimum nonzero value of radial coordinate denoted as $r_{0}$ ) and combines two asymptomatically flat regions. In order to have a proper shape of the wormhole, the shape function must attain the ratio to radial coordinate as 1 and represents increasing behavior with respect to radial coordinate which is $1-s(r) / r \geq 0$. This condition of ratio is known as flare-out condition. In addition, the value of shape function and radial coordinate must be same at throat; that is, $s\left(r_{0}\right)=r_{0}$. There are also some other constraints applied on the derivative of shape functions which must be satisfied. These are $r s^{\prime}-s<0$ and $s^{\prime}\left(r_{0}\right)<1$. Also, the proper distance $D(r)= \pm \int_{r_{0}}^{r}(1-s(r) / r)^{-1 / 2} d r$ must meet the criteria as decreasing behavior from upper region $D=+\infty$ towards throat where $d=0$ and then towards lower region where $D=-\infty$.

In order to make the wormhole traversable, the throat must remain open. To prevent shrinking of wormhole 
throat, there must exist such form of energy-momentum tensor which provides the corresponding matter content. This matter content violates the energy conditions in order to keep throat open and thus is named as exotic matter. This implies that violation of these conditions is the basic key ingredient to construct traversable wormhole solutions. Since the usual energy-momentum tensor satisfies the energy conditions, therefore the search for wormhole solutions for which violation may come from other sources while matter content satisfying energy conditions becomes one of the most challenging problems in astrophysics.

The higher dimensional gravity theories and modified theories may play positive role by providing violation from higher order Lagrangian terms and effective form of energymomentum tensor. The relationship between Raychaudhuri equation and attractiveness of gravity yields the weak energy condition (WEC) as $\mathscr{T}_{\alpha \beta} \mu^{\alpha} \mu^{\beta} \geq 0$, for any time-like vector $\mu^{\alpha}$. In terms of components of the energy-momentum tensor, this inequality yields $\rho \geq 0$ and $\rho+p \geq 0$. The null energy condition (NEC) is developed by continuity through WEC; that is, the NEC is $\mathscr{T}_{\alpha \beta} \chi^{\alpha} \chi^{\beta} \geq 0$, for any null vector $\chi^{\alpha}$. This inequality gives $\rho+p \geq 0$. Also, it is noted that WEC keeps NEC.

The anisotropic energy-momentum is given by

$$
\mathscr{T}_{\beta}^{\alpha}=\left(\rho+p_{r}\right) u^{\alpha} u_{\beta}-p_{r} g_{\beta}^{\alpha}+\left(p_{t}-p_{r}\right) \eta^{\alpha} \eta_{\beta} \text {, }
$$

where $p_{r}$ and $p_{t}$ are the radial and tangential pressure components with $\rho=\rho(r), p=p(r)$, and satisfy $u^{\alpha} u_{\beta}=$ $-\eta^{\alpha} \eta_{\beta}=1$. Using (3), the field equations become

$$
\begin{aligned}
& \rho(r)=\frac{(n-2)}{2 r^{2}}\left[\left(s^{\prime}-\frac{s}{r}\right)\left(1+\frac{2 \epsilon s}{r^{3}}\right)\right. \\
& \left.+\frac{s}{r}\left\{(n-3)+(n-5) \frac{\epsilon s}{r^{3}}\right\}\right], \\
& p_{r}(r)=\frac{(n-2)}{2 r}\left[2\left(1-\frac{s}{r}\right)\left(1+\frac{2 \epsilon s}{r^{3}}\right) \lambda^{\prime}\right. \\
& \left.-\frac{s}{r^{2}}\left\{(n-3)+(n-5) \frac{\epsilon s}{r^{3}}\right\}\right], \\
& p_{t}(r)=\left(1-\frac{s}{r}\right)\left(1+\frac{2 \epsilon s}{r^{3}}\right)\left[\lambda^{\prime \prime}+\lambda^{\prime 2}+\frac{\left(s-r s^{\prime}\right) \lambda^{\prime}}{2 r(r-s)}\right] \\
& +\left(1-\frac{s}{r}\right)\left(\frac{\lambda^{\prime}}{r}+\frac{s-r s^{\prime}}{2 r^{2}(r-s)}\right)\{(n-3) \\
& \left.+(n-5) \frac{2 \epsilon s}{r^{3}}\right\}-\frac{s}{2 r^{3}}\{(n-3)(n-4) \\
& \left.+(n-5)(n-6) \frac{\epsilon s}{r^{3}}\right\}-\frac{2 \lambda^{\prime} \epsilon}{r^{4}}\left(1-\frac{s}{r}\right)\left(s-r s^{\prime}\right)(n
\end{aligned}
$$

where prime refers derivative with respect to $r$ and $\epsilon=(n-$ $3)(n-4) \epsilon_{1}$ for the sake of notational simplicity.

\section{Wormhole Solutions}

In order to discuss the wormhole geometry and solutions, there are several frameworks and strategies used to find unknown functions. For instance, we have five unknown functions $\rho(r), p_{r}(r), p_{t}(r), \lambda(r)$, and $s(r)$ in the underlying case. One may choose some kind of equation of state representing accelerated expansion of the universe, or different forms of energy density such as energy density of static spherically symmetric object with noncommutative geometry having Gaussian or Lorentzian distributions and galactic halo region and so forth. The traceless energymomentum tensor is also used which is related to the Casimir effect. In order to construct viable wormhole solutions in $n$ dimensional Einstein-Gauss-Bonnet gravity, we assume different forms of energy density of noncommutative geometry in the following.

Nicolini et al. [21] have improved the short distance behavior of point-like structures in a new conceptual approach based on coordinate coherent state formalism to noncommutative gravity. In their method, curvature singularities which appear in general relativity can be eliminated. They have demonstrated that black hole evaporation process should be stopped when a black hole reaches a minimal mass. This minimal mass, named black hole remnant, is a result of the existence of a minimal observable length. This approach, which is the so-called noncommutative geometry inspired model, via a minimal length caused by averaging noncommutative coordinate fluctuations cures the curvature singularity in black holes. In fact, the curvature singularity at the origin of black holes is substituted for a regular de Sitter core. Accordingly, the ultimate phase of the Hawking evaporation as a novel thermodynamically steady state comprising a nonsingular behavior is concluded.

It must be noted that, generally, it is not required to consider the length scale of the coordinate noncommutativity to be the same as the Planck length. Since, the noncommutativity influences appear on a length scale connected to that region, they can behave as an adjustable parameter corresponding to that pertinent scale. The presence of a universal short distance cut-off leads to the effects such as in quantum field theory; it curves UV divergences while it cures curvature singularities in general relativity. In the specific case of the gravity field equations, the only modification occurs at the level of the energy-momentum tensor, while $G_{\mu \nu}$ is formally left unchanged. In nonommutative space, the usual definition of mass density in the form of Dirac delta function does not hold. So in this space the usual form of the energy density of the static spherically symmetry smeared and particle-like gravitational source requires some other forms of distribution.

In view of the above explanations, we are going to discuss wormhole solutions with the help of two well-known energy distributions such as Gaussian and Lorentzian in noncommutative scenario. As an important remark, the essential aspects of the noncommutativity approach are not specifically sensitive to any of these distributions of the smearing effects [22] rather only distribution parameter is different. The Gaussian source has also been used by Sushkov [23] to model 


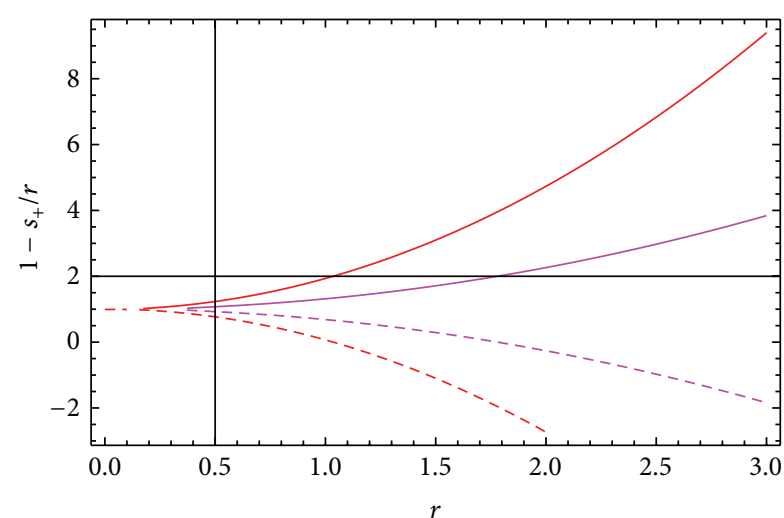

(a)

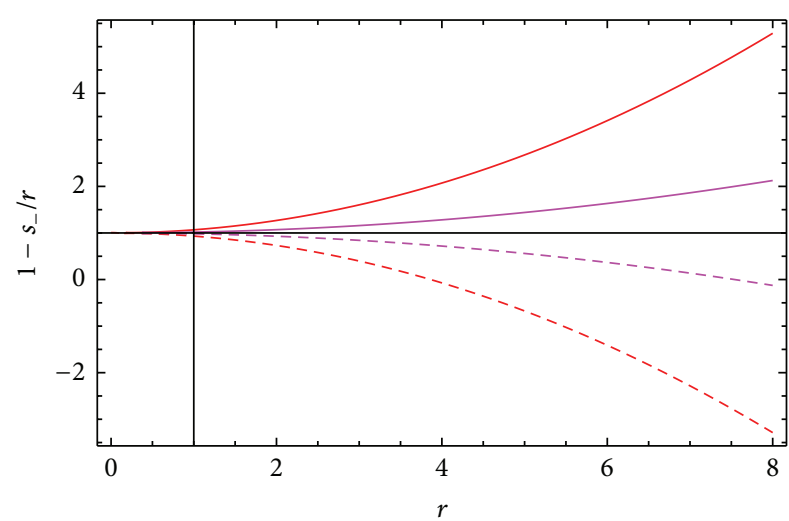

(b)

Figure 1: Plots of (a) $1-s_{+} / r$ and (b) $1-s_{-} / r$ versus $r$ in Gaussian distribution for $n=5, \epsilon=1$ (red), $n=5, \epsilon=-1$ (red dashed), $n=6, \epsilon=3$ (purple), and $n=6, \epsilon=-3$ (purple dashed).

phantom-energy supported wormholes, as well as by Nicolini and Spallucci [24] for the purpose of modeling the physical effects of short distance fluctuations of noncommutative coordinates in the study of black holes. Galactic rotation curves inspired by a noncommutative geometry background are discussed [25]. The stability of a particular class of thinshell wormholes in noncommutative geometry is analyzed elsewhere [26].

3.1. Gaussian Distributed Noncommutative Framework. An intrinsic characteristic of spacetime is the noncommutativity which plays an effective role in several areas. It is an interesting consequence of string theory where the coordinates of spacetime become noncommutative operators on $D$-brane [27]. The noncommutativity of spacetime can be converted in the commutator, $\left[x^{\alpha}, x^{\beta}\right]=i \theta^{\alpha \beta}$, where $\theta^{\alpha \beta}$ is an antisymmetric matrix describing discretization of spacetime and has dimension (length) ${ }^{2}$. This discretization process is similar to the discretization of phase space by Planck constant. Replacing the point-like structures with smeared objects, the energy density of the particle-like static spherically symmetric gravitational source having mass $M$ takes the following form [21]:

$$
\rho_{\mathrm{nc}}=\frac{M}{(4 \pi \theta)^{(n-1) / 2}} e^{-r^{2} / 4 \theta},
$$

where $\theta$ is the noncommutative parameter in Gaussian distribution. The mass $M$ could be a diffused centralized object such as a wormhole [28]. It is mentioned here that the smearing effect is achieved by replacing the Gaussian distribution of minimal length $\sqrt{\theta}$ with the Dirac delta function. In order to construct $n$-dimensional Einstein Gauss-Bonnet wormhole geometry, we equate the energy density given in (7) and $\rho_{\mathrm{nc}}$ in (10) yields the following differential equation:

$$
\begin{aligned}
s^{\prime} & =\frac{1}{1+2 s \epsilon / r^{3}}\left[\left(4-n+(7-n) \frac{s \epsilon}{r^{3}}\right)\right. \\
& \left.+\frac{2 r^{2} M}{(n-2)}(4 \pi \theta)^{(1-n) / 2} e^{-r^{2} / 4 \theta}\right] .
\end{aligned}
$$

The solution of this equation is

$$
\begin{aligned}
& s(r)=-\frac{1}{2}\left[\frac{r^{3}}{\epsilon}\right. \\
& \pm \frac{r^{(7-n) / 2} \pi^{-n / 4}}{\sqrt{\epsilon(n-1)}}\left\{(n-2) \pi^{n / 2}\left(r^{n-1}+4 \epsilon^{2} c_{1}\right)\right. \\
& \left.\left.\quad-4 M \sqrt{\pi} \epsilon \text { Gamma }\left[\frac{n-1}{2}, \frac{r^{2}}{4 \theta}\right]\right\}^{1 / 2}\right],
\end{aligned}
$$

where $c_{1}$ is an integration constant. This solution has two roots with plus and minus signs and we assign these roots as $s_{+}(r)$ and $s_{-}(r)$ solutions. In order to plot the quantities $1-s / r, \rho, \rho+p_{r}$, and $\rho+p_{t}$ to obtain wormhole solutions for both of these solutions, we restrict ourselves to five- and six-dimensional cases with constant redshift function $\lambda=0$. The expressions of NEC takes the form

$$
\begin{aligned}
\rho+p_{r}= & \frac{(n-2)}{2 r^{2}}\left(s^{\prime}-\frac{s}{r}\right)\left(1+\frac{2 \epsilon s}{r^{3}}\right), \\
\rho+p_{t}= & \frac{r s^{\prime}-s}{2 r^{3}}\left(1+\frac{6 \epsilon s}{r^{3}}\right) \\
& +\frac{s}{r^{3}}\left[(n-3)+(n-5) \frac{2 s \epsilon}{r^{3}}\right] .
\end{aligned}
$$

In this regard, we assume values of some constants as $M=0.008, \theta=0.002$ while $\epsilon_{1}=0.5,-0.5$ result in $\epsilon=$ $1,-1$ for five-dimensional and $\epsilon=3,-3$ for six-dimensional case. Figure 1(a) represents the plot of $1-s_{+} / r$ versus $r$ for five and six-dimensional wormhole solutions. The graph represents positively increasing behavior for positive $\epsilon$ for both curves. For negative $\epsilon$, we examine that graph initially represents positive behavior for both dimensions in the range $r<1$ and $r<1.8$ for $n=5$ and 6, respectively, and then decreases towards negative values. In Figure 1(b), the graph of $1-s_{-} / r$ versus $r$ shows the same behavior for all curves as in Figure 1(a), with positive behavior of dashed curves in the range $r<4$ and $r<8$ for $n=5$ ad 6 . However, we have less 


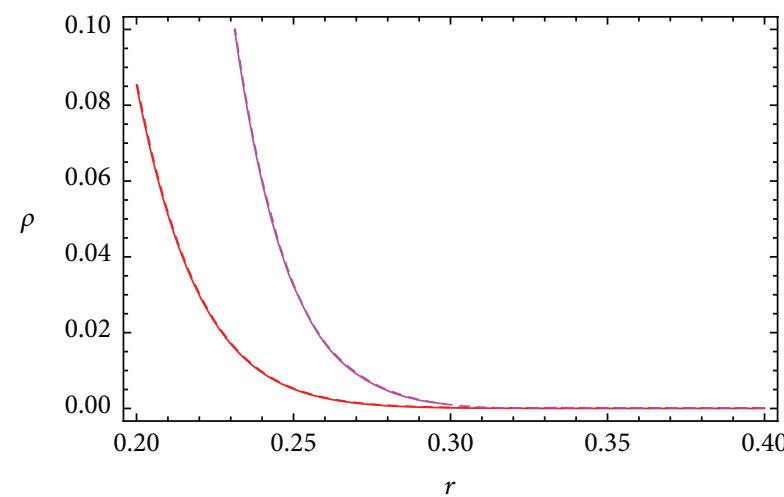

(a) Evolution of $\rho_{+}$versus $r$

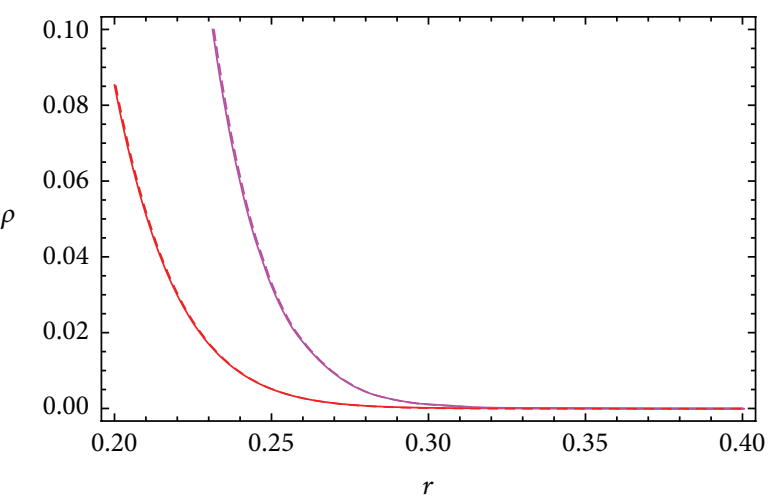

(b) Evolution of $\rho_{-}$versus $r$

Figure 2: Plots of (a) $\rho_{+}$and (b) $\rho_{-}$versus $r$ in Gaussian distribution for $n=5, \epsilon=1$ (red), $n=5, \epsilon=-1$ (red dashed), $n=6, \epsilon=3$ (purple), and $n=6, \epsilon=-3$ (purple dashed).

possibility of wormhole scenario with respect to $r$ for positive root solution as compared to negative root solution of shape function.

Figure 2 shows the behavior of energy density versus $r$ as positively decreasing behavior for both dimensions corresponding to $n=5$ and 6 dimensions. For $s_{+}$and positive $\epsilon$, the plot of $\rho+p_{r}$ represents positive behavior in decreasing manner for $n=5$ and 6 while representing negative behavior for negative $\epsilon$ as shown in Figure 3(a). In Figure 3(b), we examine same behavior for negative root solution. Figure 4(a) depicts the opposite behavior to Figure 3(a), that is, for $n=$ 5,6 dimensions and positive $\epsilon, \rho+p_{t}$ demonstrates negative behavior. Thus, these plots express the violation of WEC incorporating the case of $s_{+}$. For $s_{-}$, we obtain the same behavior as in Figure 3(b) which indicates positive behavior for positive $\epsilon$ and $n=5,6$ and negative behavior for negative $\epsilon$ and $n=5,6$. This implies that WEC is satisfied for negative root solution with positive Gauss-Bonnet coefficient. Thus, we obtain physically acceptable wormhole solutions satisfying WEC for both dimensions.

3.2. Lorentzian Distributed Non-Commutative Framework. Now we consider the case of noncommutative geometry with Lorentzian distribution. The energy density of point-like source under this distribution becomes $[8,15]$

$$
\rho_{\mathrm{Lnc}}=\frac{M \sqrt{\phi}}{\pi^{2}\left(r^{2}+\phi\right)^{n / 2}},
$$

where $\phi$ is the noncommutative parameter in Lorentzian distribution. Inserting $\rho_{\text {Lnc }}$ in (7), the differential equation takes the following form:

$$
\begin{aligned}
s^{\prime} & =\frac{1}{1+2 s \epsilon / r^{3}}\left[\frac{2 r^{2} M \sqrt{\phi}}{\pi^{2}(n-2)\left(r^{2}+\phi\right)^{n / 2}}\right. \\
& \left.+\frac{b}{r}\left\{4-n+(7-n) \frac{s \epsilon}{r^{3}}\right\}\right] .
\end{aligned}
$$

The solution of this equation is given by

$$
\begin{aligned}
& s(r)=\frac{r^{3}}{2 \epsilon \pi}\left[-\pi \pm \frac{1}{(n-2)(n-1)}\left\{\pi ^ { 2 } ( n ^ { 2 } - 3 n + 2 ) \left(r^{n}\right.\right.\right. \\
& \left.+4 r \epsilon^{2} c_{2}\right) \\
& +8 M \epsilon r^{n} \phi^{(1-n) / 2} \text { Hypergeometric } 2 \mathrm{~F} 1\left[\frac{1}{2}(n-1), \frac{n}{2}\right. \\
& \left.\left.\left.\quad \frac{n+1}{2},-\frac{r^{2}}{\phi}\right]\right\}^{1 / 2}\right],
\end{aligned}
$$

where $c_{2}$ is an integration constant. We again assign both solutions as $s_{+}$and $s_{-}$and explore the wormhole solutions. In this case, we choose constants as $\phi=2, M=2$, and $c_{2}=0.5$ for same dimensions and Gauss-Bonnet coefficient as for noncommutative background.

In Figure 5(a), we plot $1-s_{+} / r$ versus $r$ which represents that the condition for wormhole geometry, that is, $1-s / r>0$, holds for 5 and 6 dimensions with negative $\epsilon$. For positive $\epsilon$, the positivity of this expression depends on some ranges so that it remains positive for $r<1.9$ for $n=5$ while $n=6$ observes the range $r<2.1$. Figure 5(b) corresponds to the plot of $1-s_{-} / r$ with respect to $r$ showing positive behavior for positive $\epsilon$. For $n=5$, it expresses a very short range, $r<0.5$, for positivity whereas it remains negative for 6 dimensional solution. That is, we have no wormhole solution for $n=6, \epsilon=-3$ taking negative root solution. So, we skip this case in further discussion. The behavior of $\rho_{+}$and $\rho_{-}$ remains positive for all cases except $n=6, \epsilon=-3$ for which it describes negative behavior, as shown in Figures 6(a) and 6(b). This implies that we have no wormhole solutions for 6dimensional case with negative Gauss-Bonnet coefficient for both solutions.

Figure 7(a) expresses the behavior of $\rho+p_{r}$ for positive root solution versus $r$ which remains positive for the range $r<1$ for $n=5$ and 6 with positive $\epsilon$ and then turns towards negative behavior. It demonstrates negative behavior for $r<1$ and then moves to positive region. Incorporating $s_{-}$solution, $\rho+p_{r}$ shows positive behavior only for the case $n=5$, 


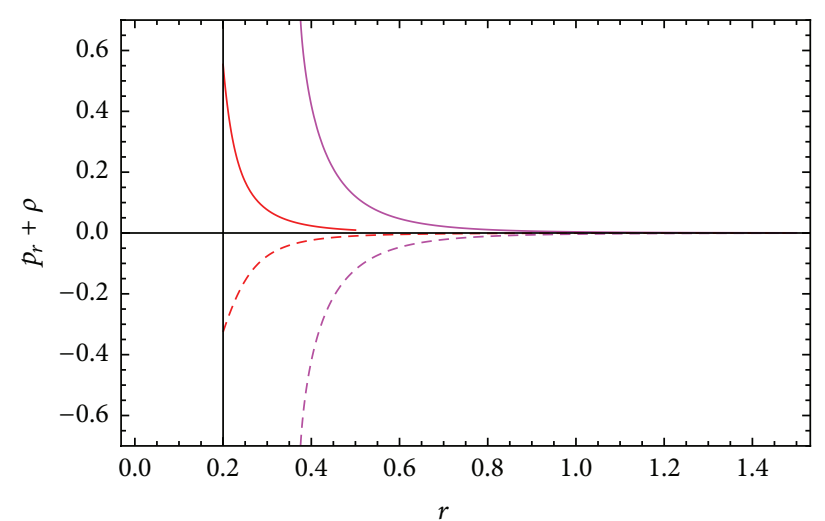

(a) Evolution of $\left(\rho+p_{r}\right)^{+}$versus $r$

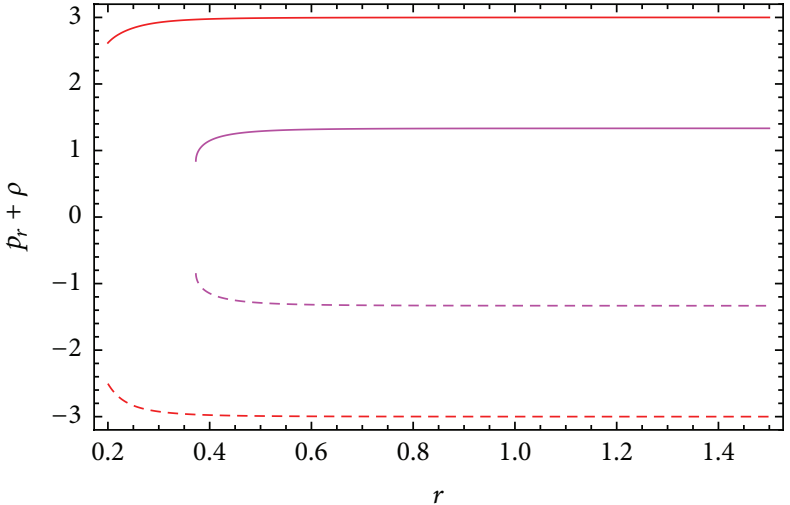

(b) Evolution of $\left(\rho+p_{r}\right)^{-}$versus $r$

Figure 3: Plots of (a) $\rho+p_{r}$ for $s_{+}$and (b) $\rho+p_{r}$ for $s_{-}$versus $r$ in Gaussian distribution for $n=5, \epsilon=1$ (red), $n=5, \epsilon=-1$ (red dashed), $n=6, \epsilon=3$ (purple), and $n=6, \epsilon=-3$ (purple dashed).

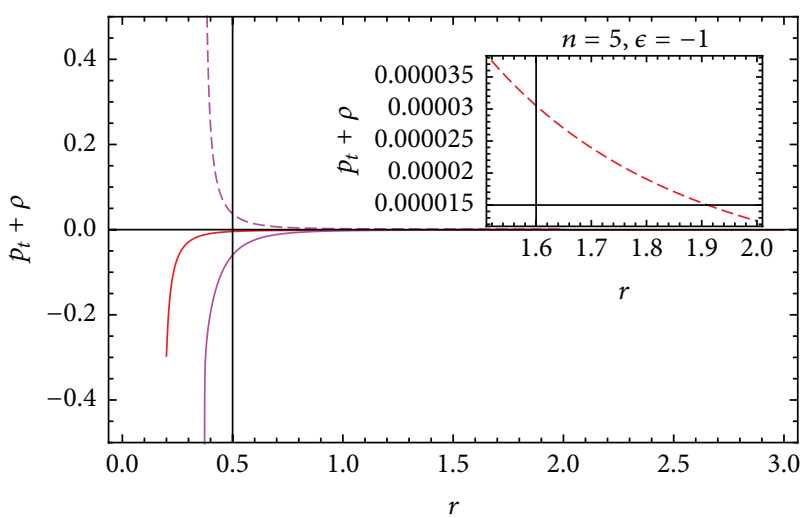

(a) Evolution of $\left(\rho+p_{t}\right)^{+}$versus $r$

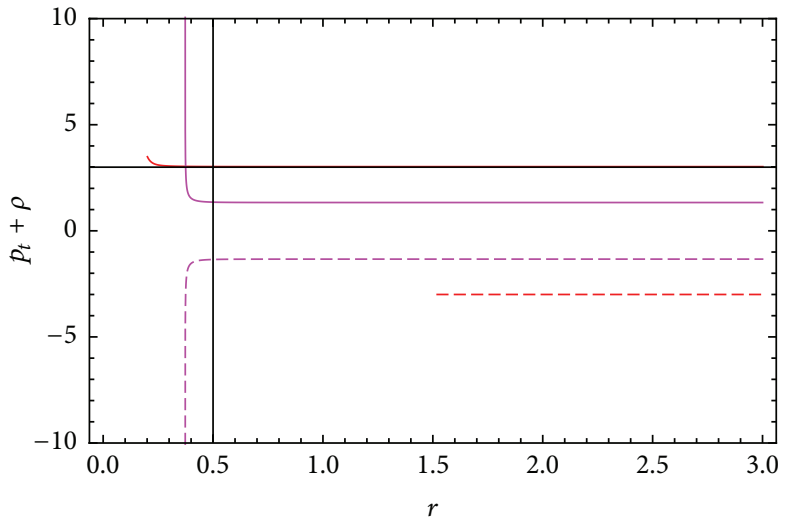

(b) Evolution of $\left(\rho+p_{t}\right)^{-}$versus $r$

Figure 4: Plots of (a) $\rho+p_{t}$ for $s_{+}$and (b) $\rho+p_{t}$ for $s_{-}$versus $r$ for in Gaussian distribution $n=5, \epsilon=1$ (red), $n=5, \epsilon=-1$ (red dashed), $n=6, \epsilon=3$ (purple), and $n=6, \epsilon=-3$ (purple dashed).

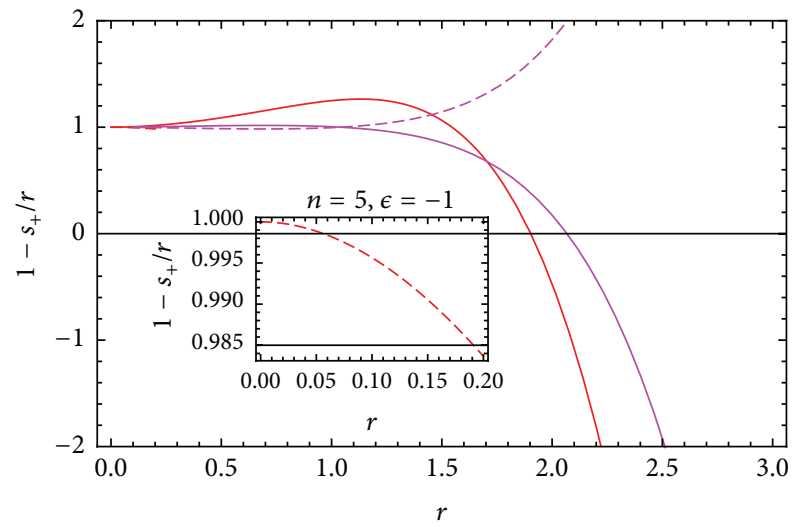

(a)

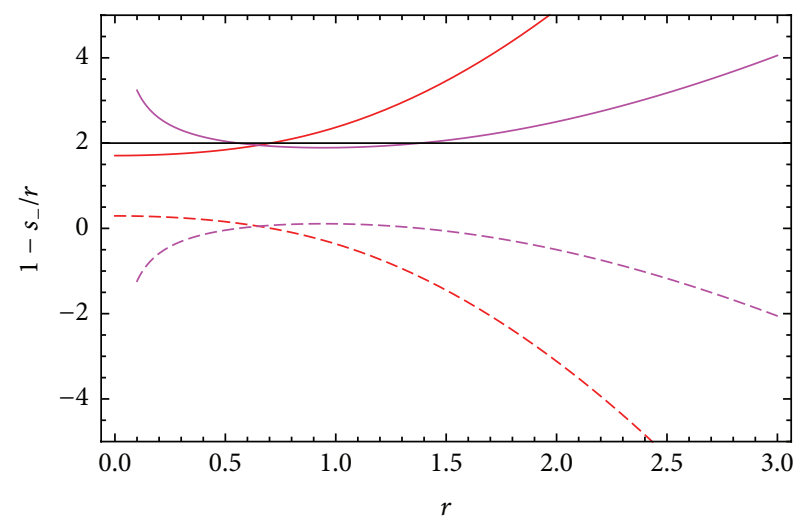

(b)

Figure 5: Plots of (a) $1-s_{+} / r$ and (b) $1-s_{-} / r$ versus $r$ in Lorentzian distribution for $n=5, \epsilon=1$ (red), $n=5, \epsilon=-1$ (red dashed), and $n=6$, $\epsilon=3$ (purple). 


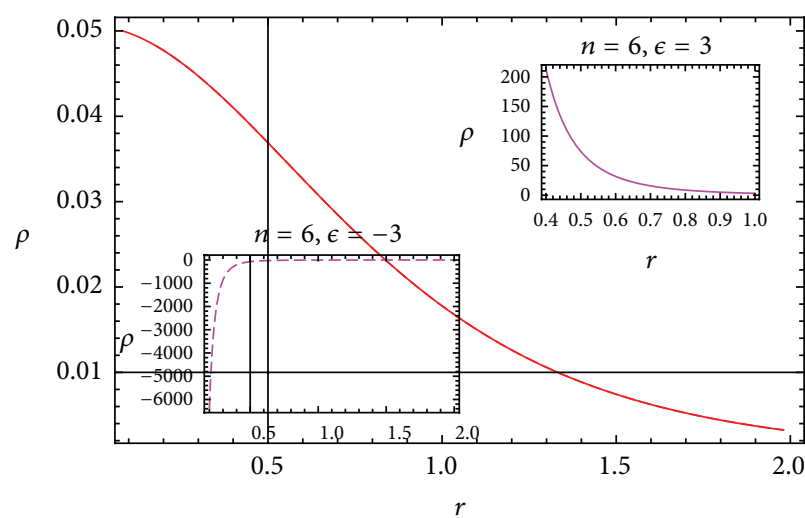

(a) Evolution of $\rho_{+}$versus $r$

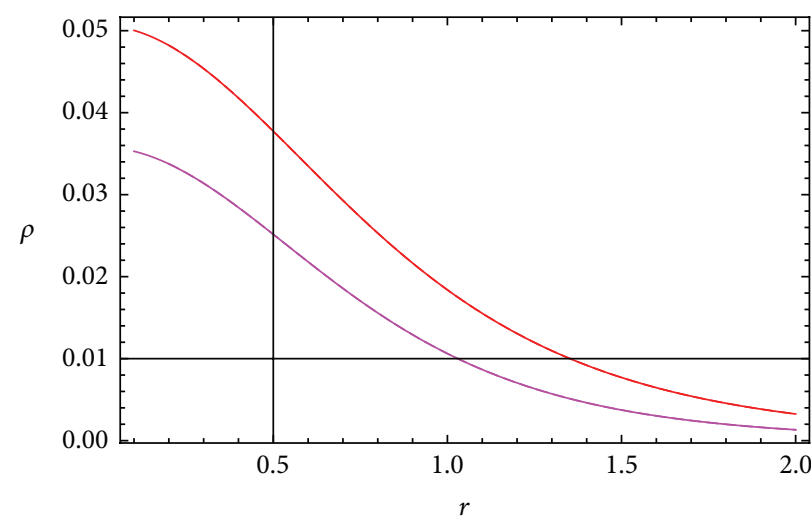

(b) Evolution of $\rho_{-}$versus $r$

Figure 6: Plots of (a) $\rho_{+}$and (b) $\rho_{-}$versus $r$ in Lorentzian distribution for $n=5, \epsilon=1$ (red), $n=5, \epsilon=-1$ (red dashed), and $n=6, \epsilon=3$ (purple). For (a) $\rho_{+}, n=6$, and $\epsilon=-3$ (purple dashed).

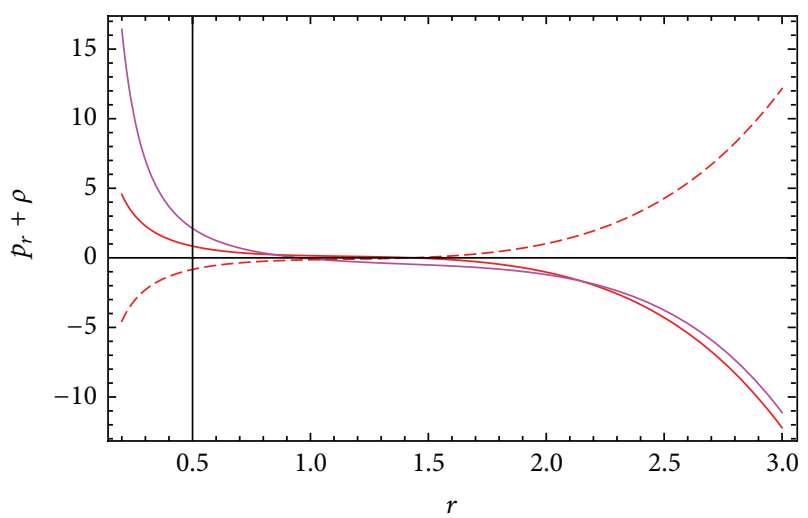

(a) Evolution of $\left(\rho+p_{r}\right)_{+}$versus $r$

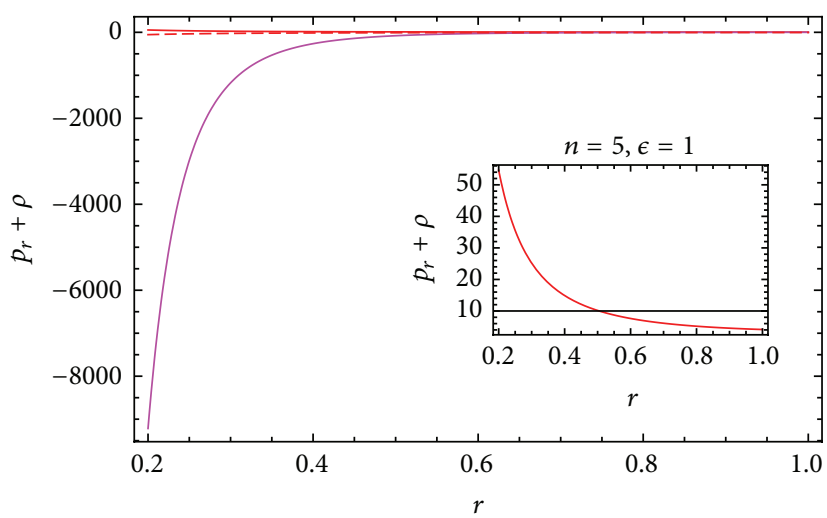

(b) Evolution of $\left(\rho+p_{r}\right)_{-}$versus $r$

Figure 7: Plots of (a) $\rho+p_{r}$ for $s_{+}$and (b) $\rho+p_{r}$ for $s_{-}$versus $r$ in Lorentzian distribution for $n=5, \epsilon=1$ (red), $n=5, \epsilon=-1$ (red dashed), and $n=6, \epsilon=3$ (purple).

$\epsilon>0$ and negative behavior for the remaining two cases as shown in Figure 7(b). In Figures 8(a) and 8(b), we draw $\rho+p_{t}$ for both solutions versus $r$. This expression represents the negative behavior for $(n=5, \epsilon=1),(n=5, \epsilon=-1)$ with $s_{+}$and $(n=5, \epsilon=-1)$ with $s_{-}$and preserves positivity for $(n=6, \epsilon=3)$ with $s_{+},(n=5, \epsilon=1),(n=6, \epsilon=3)$ with $s_{-}$ solution.

\section{Equilibrium Condition}

In order to find the equilibrium configuration of the wormhole solutions in Gaussian as well as Lorentzian distributed noncommutative backgrounds, we use the generalized Tolman-Oppenheimer-Volkoff equation. This equation is derived by solving the Einstein equations for a general timeinvariant, spherically symmetric metric having metric tensor $g_{\alpha \beta}=\left(e^{\tau(r)},-e^{\omega(r)},-r^{2},-r^{2} \sin ^{2} \theta\right)$, where $\alpha \beta$ represents only diagonal entries and $\tau, \omega$ are general metric functions dependent on $r$. The generalized Tolman-OppenheimerVolkoff equation is

$$
\frac{d p_{r}}{d r}+\frac{\tau^{\prime}}{2}\left(\rho+p_{r}\right)+\frac{2}{r}\left(p_{r}-p_{t}\right)=0
$$

Keeping in mind the above equation, de Leon [28] proposed an equation for anisotropic mass distribution which naturally gives the equilibrium for the wormhole subject. It is given by

$$
\frac{2}{r}\left(p_{t}-p_{r}\right)-\frac{e^{(\omega-\tau) / 2} m_{\mathrm{eff}}}{r^{2}}\left(\rho+p_{r}\right)-\frac{d p_{r}}{d r}=0,
$$

where effective gravitational mass $m_{\text {eff }}=(1 / 2) r^{2} e^{(\tau-\omega) / 2} \tau^{\prime}$ is measured from throat to some arbitrary radius $r$. Accordingly, the gravitational, hydrostatic, and anisotropic force due to anisotropic matter distribution are defied as follows:

$$
\begin{aligned}
& f_{g}=-\frac{\tau^{\prime}\left(\rho+p_{r}\right)}{2}, \\
& f_{h}=-\frac{d p_{r}}{d r} \\
& f_{a}=\frac{2\left(p_{t}-p_{r}\right)}{r} .
\end{aligned}
$$

It is required that $f_{g}+f_{h}+f_{a}=0$ must hold for the wormhole solutions to be in equilibrium. In the underlying cases, we 


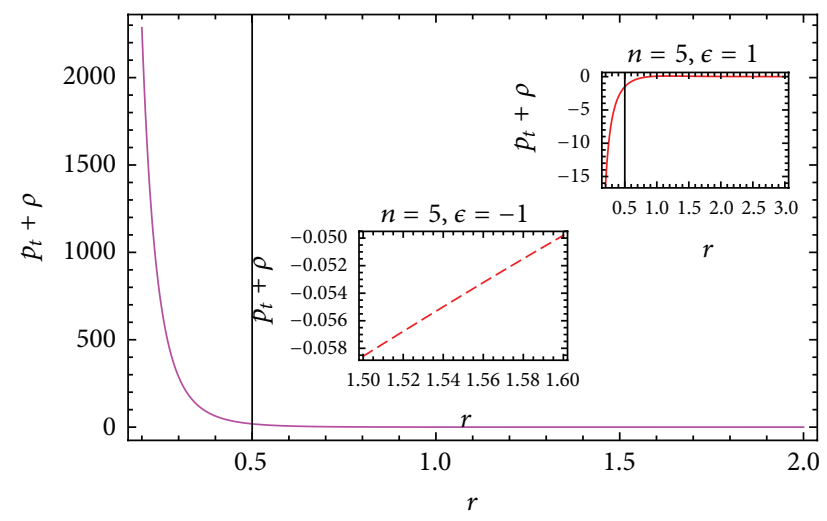

(a) Evolution of $\left(\rho+p_{t}\right)_{+}$versus $r$

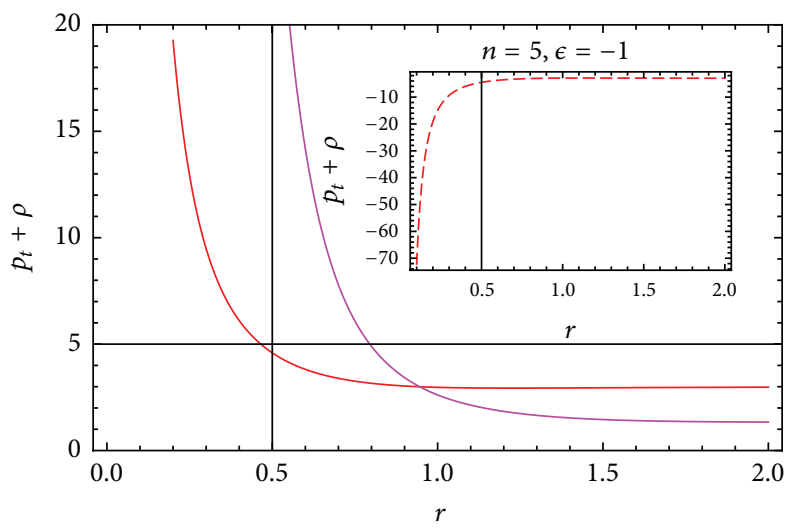

(b) Evolution of $\left(\rho+p_{t}\right)_{-}$versus $r$

Figure 8: Plots of (a) $\rho+p_{t}$ for $s_{+}$and (b) $\rho+p_{t}$ for $s_{-}$versus $r$ in Lorentzian distribution for $n=5, \epsilon=1$ (red), $n=5, \epsilon=-1$ (red dashed), and $n=6, \epsilon=3$ (purple).

proceed with constant redshift function $\lambda=0$ which vanishes the gravitational contribution $f_{g}$ in the equilibrium equation; that is, $\tau=2 \lambda$ leads to $\tau^{\prime}=0$ for constant $\lambda$. Thus, we are left with hydrostatic and anisotropic forces with corresponding equilibrium condition as

$$
f_{a}+f_{h}=0
$$

Using (8) and (9), we obtain the following expressions for the hydrostatic and anisotropic forces:

$$
\begin{aligned}
f_{a} & =\frac{1}{r^{3}}\left\{(n-1) \frac{s}{r}-s^{\prime}\right\}\left(n-3+(n-5) \frac{s \epsilon}{r^{3}}\right) \\
& -\frac{s}{r^{4}}\left[(n-3)(n-4)+(n-5)(n-6) \frac{s \epsilon}{r^{3}}\right], \\
f_{h} & =\frac{(n-2)}{2 r^{3}}\left[n-3+(n-5) \frac{s \epsilon}{r^{3}}+\frac{s \epsilon(n-5)}{r^{3}}\right] \\
& \cdot\left(s^{\prime}-\frac{3 s}{r}\right) .
\end{aligned}
$$

Figure 9 represents the plots of anisotropic as well as hydrostatic forces for the wormhole solutions in Gaussian distributed framework. Figures 9(a) and 9(c) describe the equilibrium for positive root solution for $n=5, \epsilon=$ $1,-1$ and $n=6, \epsilon=3,-3$ through opposite behavior and hence they cancel each other in order to satisfy (20). For negative root solution, $s_{-}$, Figures 9 (b) and 9 (d) show the stable configuration of wormhole solutions for fifth dimensional case only. The behaviors of both forces (which are not in opposite manner) do not cancel each other so there is no equilibrium configuration examined for sixth dimensional wormhole solutions. In the case of Lorentzian noncommutative background, Figure 10 expresses that all wormhole solutions are in equilibrium by satisfying the equilibrium condition for both dimensions.
TABLE 1: Wormhole solutions with Gaussian distributed noncommutative framework for $s_{+}$.

\begin{tabular}{lcccc}
\hline Expressions & $n=5, \epsilon=$ & $n=5, \epsilon=$ & $n=6, \epsilon=$ & $n=6, \epsilon=$ \\
& 1 & -1 & 3 & -3 \\
\hline $1-s_{+} / r$ & Positive & Positive & Positive & Positive \\
& for $r<1$ & & \\
$\rho$ & Positive & Positive & Positive & Positive \\
$\rho+p_{r}$ & Positive & Negative & Positive & Negative \\
$\rho+p_{t}$ & Negative & Positive & Negative & Positive \\
WEC & Violates & Violates & Violates & Violates \\
E.C & Holds & Holds & Holds & Holds \\
\hline
\end{tabular}

\section{Conclusion}

It is a well-known fact that the existence of wormhole solutions is based on violation of NEC. Since the normal matter satisfies the energy conditions this violation is associated with an energy-momentum tensor which provides exotic matter, a hypothetical form of matter. To explore realistic model or physically acceptable wormhole solutions, it is necessary to find such a source which gives the violation of NEC while normal matter meets the energy conditions. Here in this paper, we have explored wormhole solutions in $n$-dimensional Einstein Gauss-Bonnet gravity with Gaussian and Lorentzian noncommutative backgrounds. We have restricted ourselves to fifth and sixth dimensional cases with positive as well as negative Gauss-Bonnet coefficient. Also, we have checked the condition of equilibrium for the wormhole solutions.

The results are summarized in Tables 1 and 2 . It is noted that the $s_{+}$(Tables 1 and 3 ) and $s_{-}$(Tables 2 and 4) represent the two roots of the solutions in both backgrounds and EC denotes the equilibrium condition.

In the Lorentzan distributed noncommutative framework, we have found negative energy density for $s_{+}$while 


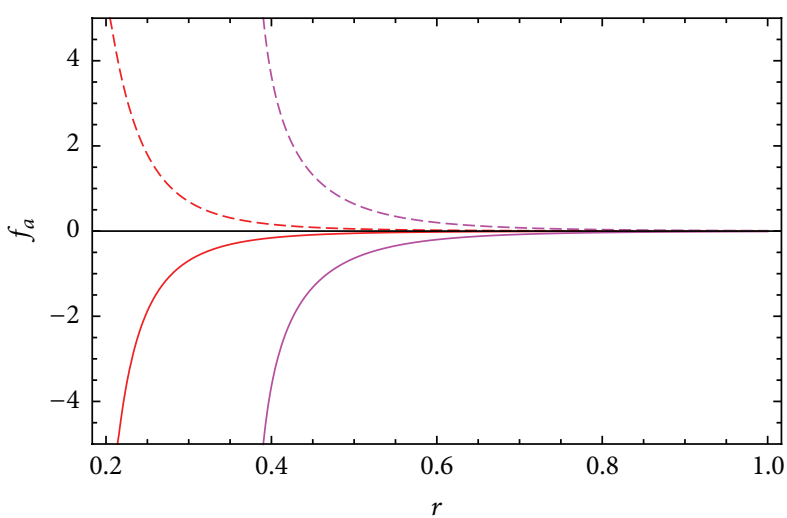

(a) Evolution of $f_{a}$ versus $r$

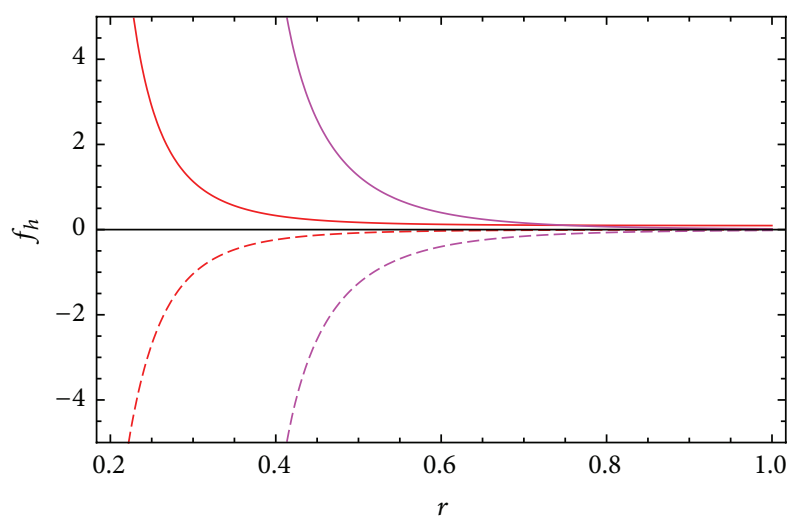

(c) Evolution of $f_{h}$ versus $r$

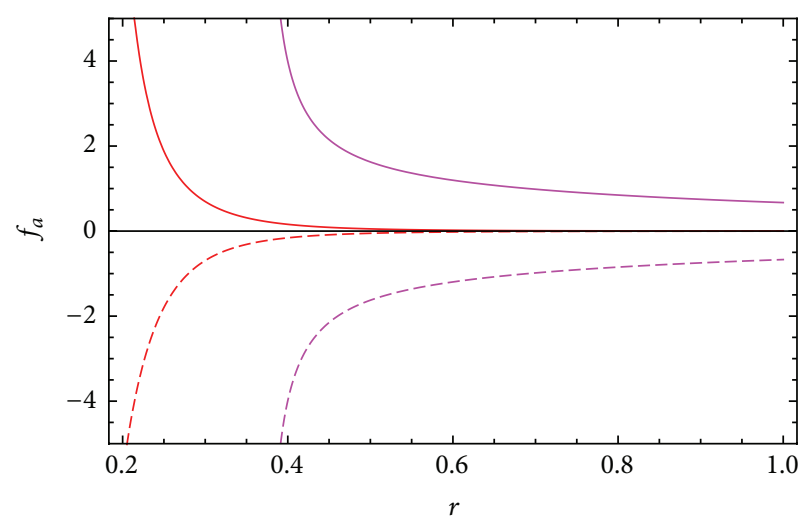

(b) Evolution of $f_{a}$ versus $r$

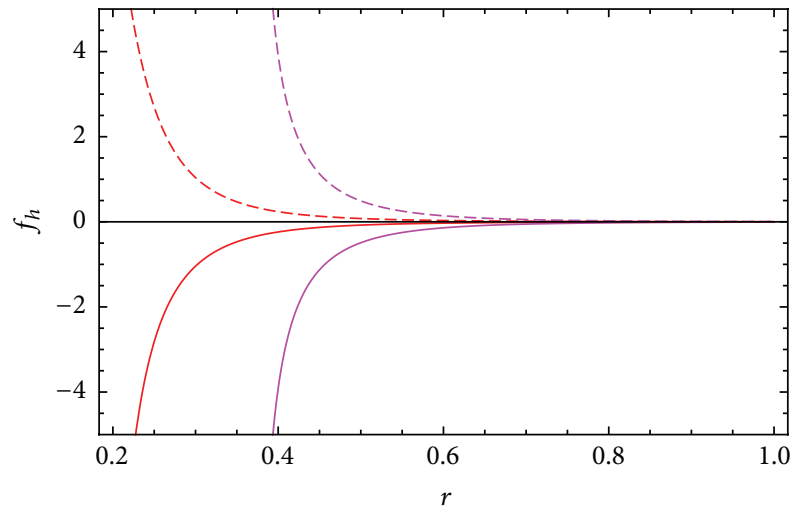

(d) Evolution of $f_{h}$ versus $r$

Figure 9: Plots of (a) $f_{a}$ for $s_{+}$, (b) $f_{a}$ for $s_{-}$, (c) $f_{h}$ for $s_{+}$, (d) $f_{h}$ for $s_{-}$versus $r$ with Gaussian distribution for $n=5, \epsilon=1$ (red), $n=5, \epsilon=-1$ (red dashed), and $n=6, \epsilon=3$ (purple).

TABLE 2: Wormhole solutions with Gaussian distributed noncommutative framework for $s_{-}$.

\begin{tabular}{|c|c|c|c|c|}
\hline Expressions & $\begin{array}{c}n=5, \epsilon= \\
1\end{array}$ & $\begin{array}{c}n=5, \epsilon= \\
-1\end{array}$ & $\begin{array}{c}n=6, \epsilon= \\
3\end{array}$ & $\begin{array}{c}n=6, \epsilon= \\
-3\end{array}$ \\
\hline $1-s_{-} / r$ & Positive & $\begin{array}{l}\text { Positive } \\
\text { for } r<4\end{array}$ & Positive & $\begin{array}{l}\text { Positive } \\
\text { for } r<8\end{array}$ \\
\hline$\rho$ & Positive & Positive & Positive & Positive \\
\hline$\rho+p_{r}$ & Positive & Negative & Positive & Negative \\
\hline$\rho+p_{t}$ & Positive & Negative & Positive & Negative \\
\hline WEC & Holds & Violates & Holds & Violates \\
\hline E.C & Holds & Holds & $\begin{array}{c}\text { Does not } \\
\text { hold }\end{array}$ & $\begin{array}{c}\text { Does not } \\
\text { hold }\end{array}$ \\
\hline
\end{tabular}

violation of $1-s / r>0$ incorporating $s_{-}$for the case $n=6$, $\epsilon=-3$ so we skipped this case. The remaining results are summarized in Tables 3 and 4.

In the paper [20], higher dimensional asymptotically flat wormhole solutions have been explored in the framework of Gauss-Bonnet gravity by considering a specific choice for a radial dependent redshift function and by imposing a particular equation of state. The WEC is satisfied at the throat by considering a negative Gauss-Bonnet coupling
TABLE 3: Wormhole solutions with Lorentzian noncommutative background for $s_{+}$.

\begin{tabular}{lccc}
\hline Expressions & $n=5, \epsilon=1$ & $n=5, \epsilon=-1$ & $n=6, \epsilon=3$ \\
\hline \multirow{2}{*}{$1-s_{+} / r$} & Positive for & Positive & Positive for \\
$\rho$ & $r<1.9$ & & $r<2.1$ \\
$\rho+p_{r}$ & Positive & Positive & Positive \\
& Positive for & Positive for & Positive for \\
$\rho+p_{t}$ & $r<1$ & $r>1$ & $r<1$ \\
WEC & Negative & Negative & Positive \\
EC & Does not & Does not & Holds for \\
& hold & hold & $r<1$ \\
& Holds & Holds & Holds \\
\hline
\end{tabular}

constant. Furthermore, they have considered a constant redshift function and shown specifically that, for negative Gauss-Bonnet coupling constant, one may have normal matter in a determined radial region and that the increase of coupling constant enlarges the normal matter region. In the present paper, we have taken energy density under noncommutative geometry distributions instead of particular equation of state. We have obtained results for positive GaussBonnet coefficient satisfying energy conditions. It contains 


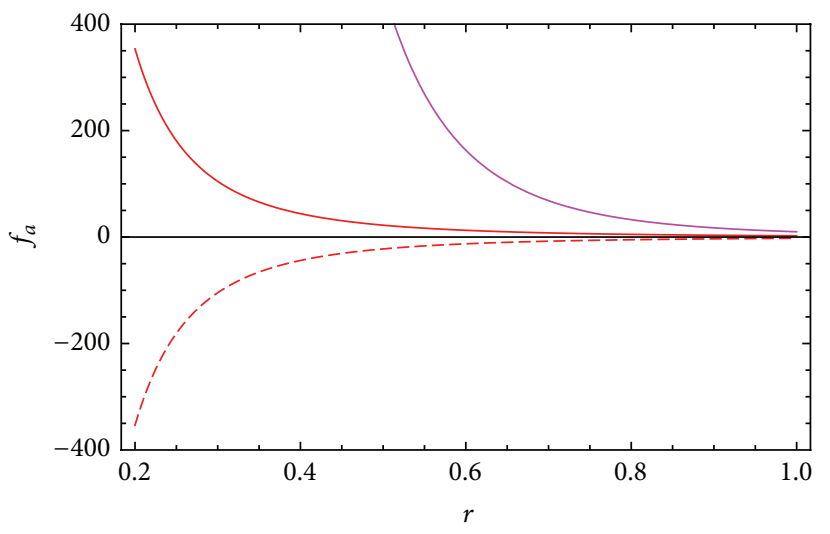

(a) Evolution of $f_{a}$ versus $r$

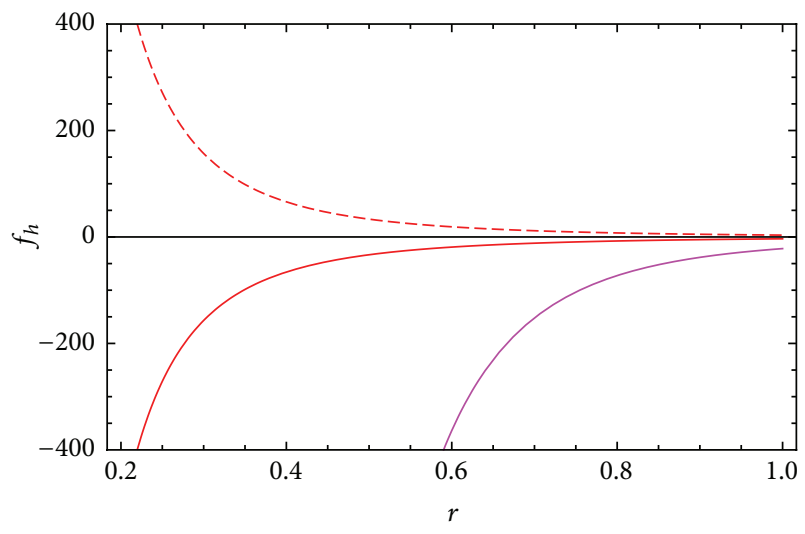

(c) Evolution of $f_{h}$ versus $r$

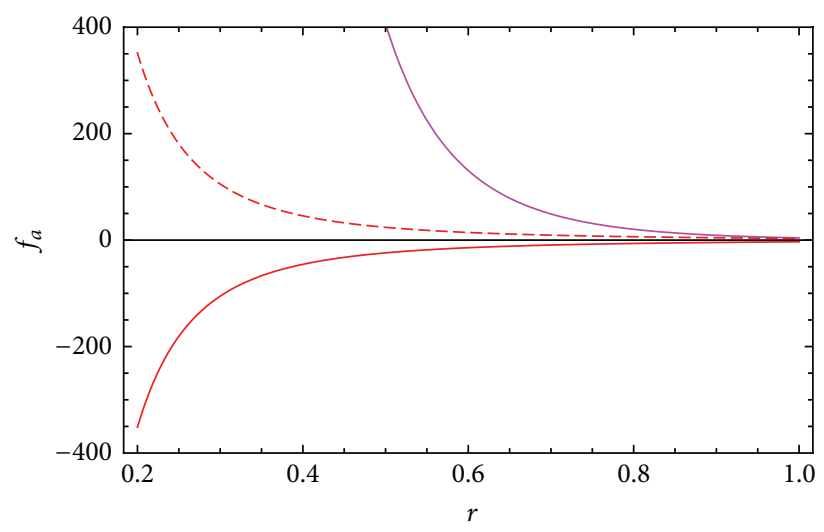

(b) Evolution of $f_{a}$ versus $r$

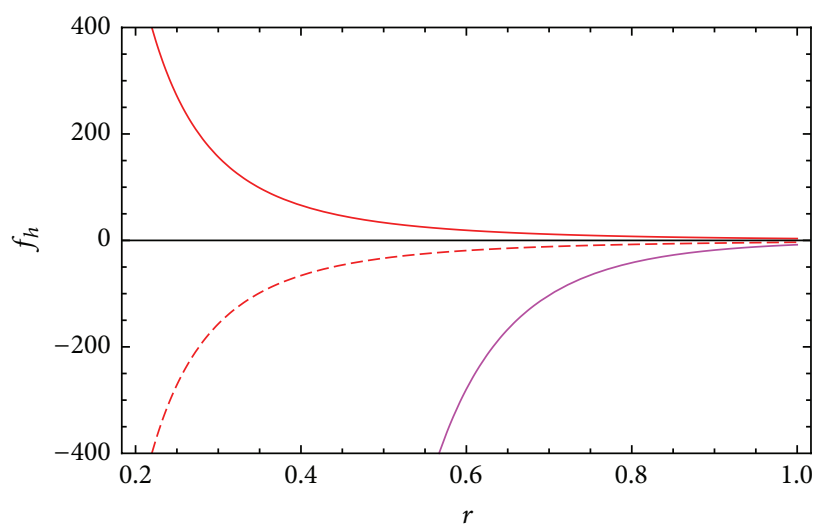

(d) Evolution of $f_{h}$ versus $r$

Figure 10: Plots of (a) $f_{a}$ for $s_{+}$, (b) $f_{a}$ for $s_{-}$, (c) $f_{h}$ for $s_{+}$, (d) $f_{h}$ for $s_{-}$versus $r$ with Lorentzian distribution for $n=5, \epsilon=1$ (red), $n=5$, $\epsilon=-1$ (red dashed), and $n=6, \epsilon=3$ (purple).

TABLE 4: Wormhole solutions with Lorentzian noncommutative background for $s_{-}$.

\begin{tabular}{lccc}
\hline Expressions & $n=5, \epsilon=1$ & $n=5, \epsilon=-1$ & $n=6, \epsilon=3$ \\
\hline $1-s_{-} / r$ & Positive & $\begin{array}{c}\text { Positive for } \\
r<0.5\end{array}$ & Positive \\
$\rho$ & Positive & Positive & Positive \\
$\rho+p_{r}$ & Positive & Negative & Negative \\
$\rho+p_{t}$ & Positive & Negative & Positive \\
WEC & Holds & Does not & Does not \\
E.C & Holds & Hold & hold \\
\hline
\end{tabular}

fifth dimensional wormhole solutions in both backgrounds satisfying equilibrium condition and sixth dimensional with disequilibrium in noncommutative background with $s_{-}$solution. Also, there is possibility for the existence of wormhole in equilibrium satisfying WEC for $n=6, \epsilon=3$ taking into account $s_{+}$solution for the range $r<1$.

\section{Conflict of Interests}

The authors declare that there is no conflict of interests regarding the publication of this paper.

\section{References}

[1] M. S. Morris and K. S. Thorne, "Wormholes in spacetime and their use for interstellar travel: a tool for teaching general relativity," American Journal of Physics, vol. 56, no. 5, pp. 395412, 1988.

[2] M. Sharif and A. Jawad, "Phantom-like generalized cosmic chaplygin gas and traversable wormhole solutions," The European Physical Journal Plus, vol. 129, article 15, 2014.

[3] F. S. N. Lobo, F. Parsaei, and N. Riazi, "New asymptotically flat phantom wormhole solutions," Physical Review D, vol. 87, no. 8, Article ID 084030, 2013.

[4] S.-W. Kim and H. Lee, "Exact solutions of a charged wormhole," Physical Review D, vol. 63, no. 6, Article ID 064014, 5 pages, 2001.

[5] M. Jamil and M. U. Farooq, "Phantom wormholes in $(2+1)$ dimensions," International Journal of Theoretical Physics, vol. 49, no. 4, pp. 835-841, 2010.

[6] M. U. Farooq, M. Akbar, and M. Jamil, "Dynamics and thermodynamics of $(2+1)$-dimensional evolving lorentzian wormhole," in Proceedings of the 3rd Algerian Workshop on Astronomy and Astrophysics, vol. 1295 of AIP Conference Proceedings, pp. 176-190, Constantine, Algeria, June 2010.

[7] P. K. Kuhfittig, "Gravitational lensing of wormholes in the galactic halo region," The European Physical Journal C, vol. 74, no. 3, article 2818, 2014. 
[8] F. Rahaman, A. Banerjee, M. Jamil, A. K. Yadav, and H. Idris, "Noncommutative wormholes in $f(R)$ gravity with lorentzian distribution," International Journal of Theoretical Physics, vol. 53, no. 6, pp. 1910-1919, 2014.

[9] M. Sharif and S. Rani, "Dynamical wormhole solutions in $f(T)$ gravity," General Relativity and Gravitation, vol. 45, no. 11, pp. 2389-2402, 2013.

[10] M. Sharif and S. Rani, "Charged noncommutative wormhole solutions in $f(T)$ gravity," The European Physical Journal Plus, vol. 129, no. 10, article 237, 2014.

[11] M. Sharif and S. Rani, "Galactic halo wormhole solutions in $f(T)$ gravity," Advances in High Energy Physics, vol. 2014, Article ID 691497, 9 pages, 2014.

[12] F. S. N. Lobo and M. A. Oliveira, "Wormhole geometries in $f(R)$ modified theories of gravity," Physical Review D, vol. 80, Article ID 104012, 2009.

[13] M. Sharif and S. Rani, "Wormhole solutions in $f(T)$ gravity with noncommutative," Physical Review D, vol. 88, no. 12, Article ID 123501, 2013.

[14] F. Rahaman, S. Islam, P. K. F. Kuhfittig, and S. Ray, "Searching for higher-dimensional wormholes with noncommutative geometry," Physical Review D, vol. 86, no. 10, Article ID 106010, 2012.

[15] P. Bhar and F. Rahaman, "Search of wormholes in different dimensional non-commutative inspired space-times with Lorentzian distribution," The European Physical Journal C, vol. 74, no. 12, article 3213, 2014.

[16] F. Rahaman, S. Karmakar, I. Karar, and S. Ray, "Wormhole inspired by non-commutative geometry," Physics Letters B, vol. 746, pp. 73-78, 2015.

[17] M. Jamil, F. Rahaman, R. Myrzakulov, P. Kuhfittig, N. Ahmed, and U. Mondal, "Nonommutative wormholes in $f(R)$ gravity," Journal of the Korean Physical Society, vol. 65, no. 6, pp. 917-925, 2014.

[18] A. Jawad and S. Rani, "Lorentz distributed noncommutative wormhole solutions in extended teleparallel gravity," The European Physical Journal C, vol. 75, article 173, 2015.

[19] B. Bhawal and S. Kar, "Lorentzian wormholes in EinsteinGauss-Bonnet theory," Physical Review D, vol. 46, no. 6, pp. 2464-2468, 1992.

[20] M. R. Mehdizadeh, M. K. Zangeneh, and F. S. N. Lobo, "Einstein-Gauss-Bonnet traversable wormholes satisfying the weak energy condition," Physical Review D, vol. 91, no. 8, Article ID 084004, 2015.

[21] P. Nicolini, A. Smailagic, and E. Spallucci, "Noncommutative geometry inspired Schwarzschild black hole," Physics Letters B, vol. 632, no. 4, pp. 547-551, 2006.

[22] S. H. Mehdipour, "Entropic force approach to noncommutative Schwarzschild black holes signals a failure of current physical ideas," The European Physical Journal Plus, vol. 127, article 80, 2012.

[23] S. Sushkov, "Wormholes supported by a phantom energy," Physical Review D, vol. 71, no. 4, Article ID 043520, 2005.

[24] P. Nicolini and E. Spallucci, "Noncommutative geometryinspired dirty black holes," Classical and Quantum Gravity, vol. 27, no. 1, Article ID 015010, 2010.

[25] F. Rahaman, P. K. Kuhfittig, K. Chakraborty, A. A. Usmani, and S. Ray, "Galactic rotation curves inspired by a noncommutativegeometry background," General Relativity and Gravitation, vol. 44, no. 4, pp. 905-916, 2012.
[26] P. K. F. Kuhfittig, "On the stability of thin-shell wormholes in noncommutative geometry," Advances in High Energy Physics, vol. 2012, Article ID 462493, 12 pages, 2012.

[27] N. Seiberg and E. Witten, "String theory and noncommutative geometry," Journal of High Energy Physics, vol. 1999, no. 9, article 032, 1999.

[28] J. P. de Leon, "Mass and charge in brane-world and noncompact Kaluza-KLEin theories in 5 dim," General Relativity and Gravitation, vol. 35, no. 8, pp. 1365-1384, 2003. 

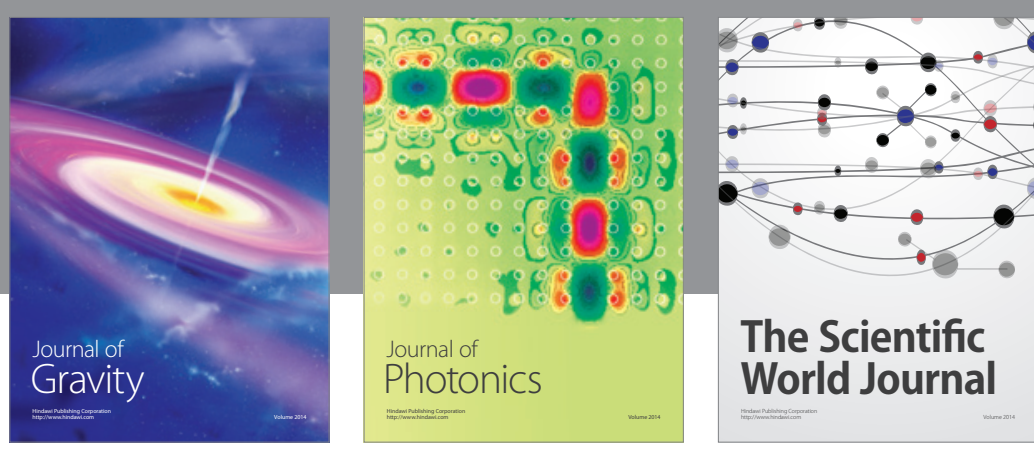

The Scientific World Journal
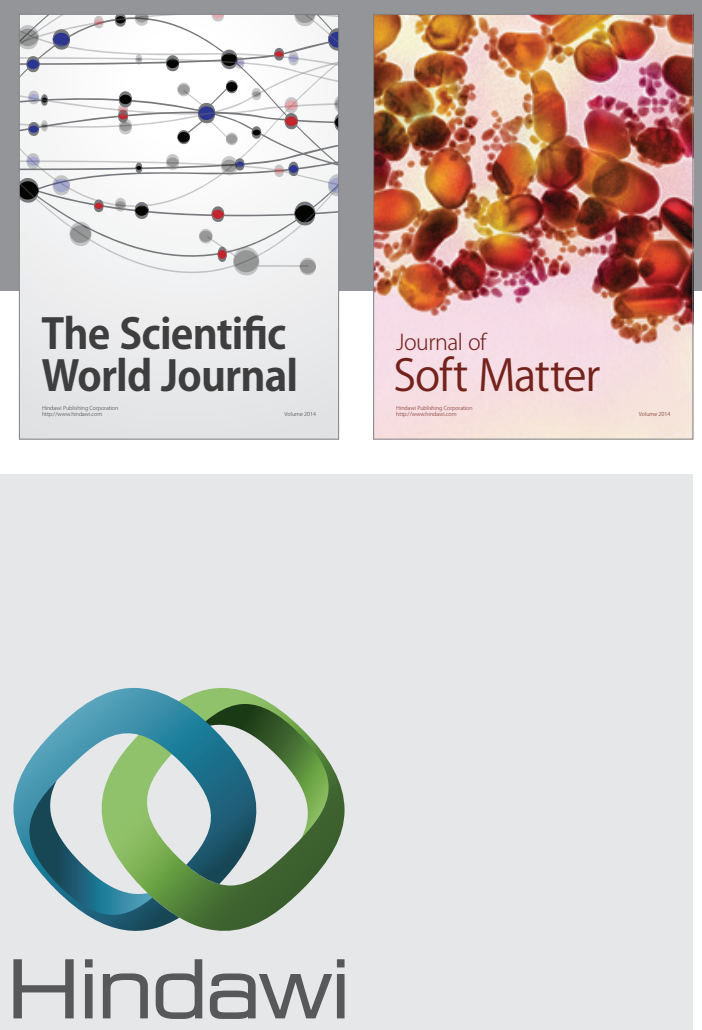

Submit your manuscripts at

http://www.hindawi.com

nternational Journal of

Statistical Mechanics
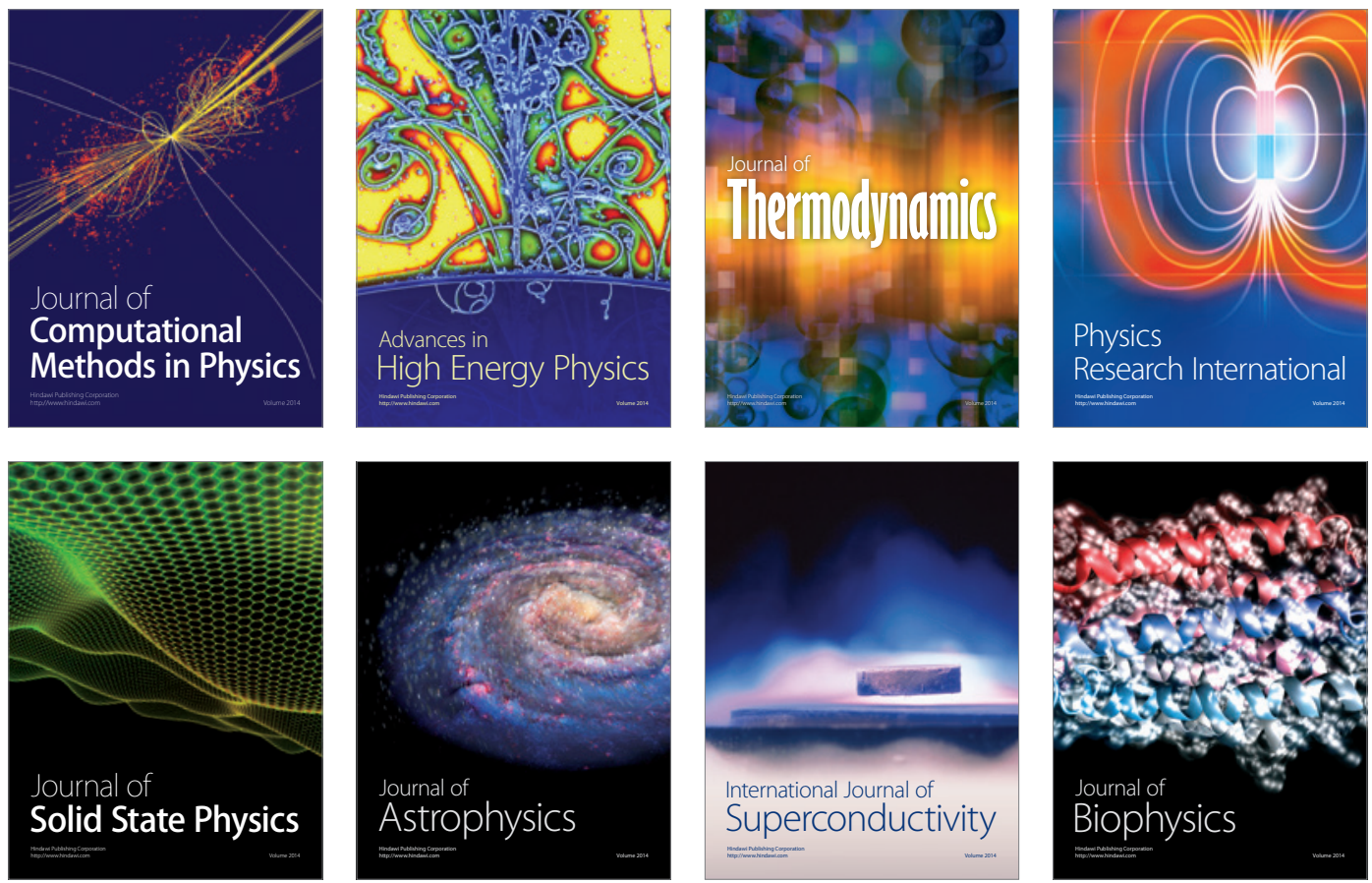
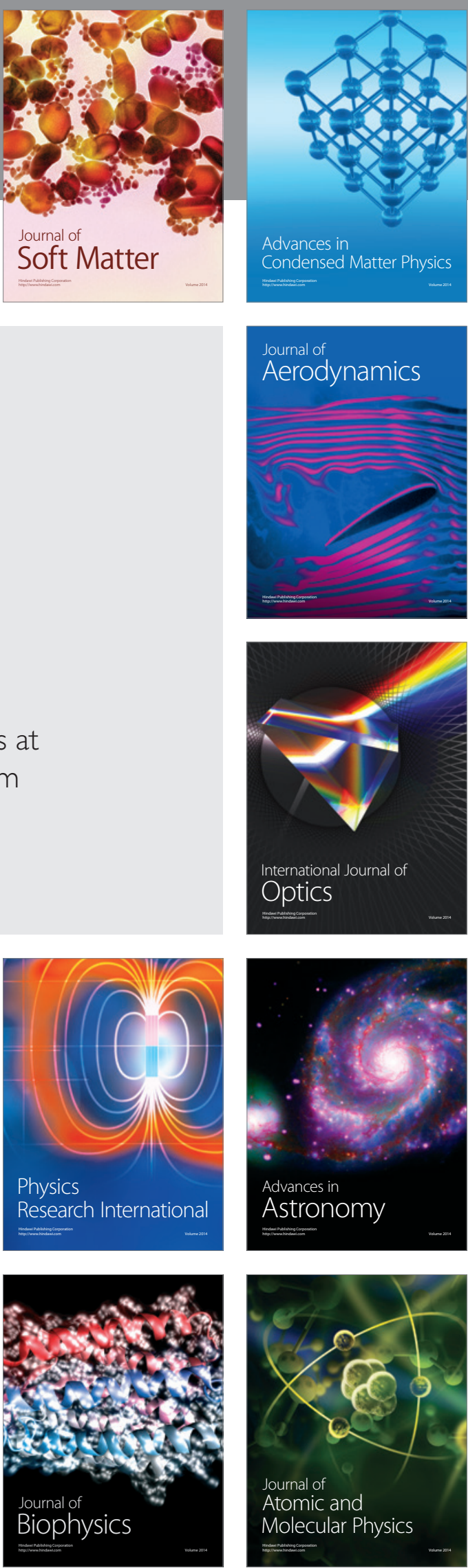